\title{
Your data are not a product
}

\begin{abstract}
The Portable Legal Consent for Common Genomics Research (PLC-CGR) is an experimental bioethics protocol that provides maximum utility to researchers who agree to its terms and protection for the de-identified personal and genomic data volunteered by informed research subjects. Data and resulting publications from this protocol are equally available to all academic, nonprofit and commercial competitors, so that intellectual property claims should arise only on new discoveries based on the data.
\end{abstract}

$\mathrm{P}$ reclinical research on human subjects proceeds far too slowly and is successful far too infrequently. One reason for this is that existing data sets generated to answer research questions in one setting are unavailable for reuse by other researchers, according to the terms of the informed consent agreements under which the data were originally obtained from research subjects. More often, data access is restricted by data holders for proprietary reasons or even for precautionary reasons because of uncertainty about legal and ethical positions. It is true that there are now projects in which detailed genomic and medical data have been obtained from informed volunteers who allow full public release of the data, as in the example of the Personal Genome Project (http://www.personal genomes.org/). However, such highly informed and altruistic volunteers are not representative of the population as a whole. Most people are willing to share their data only in exchange for detailed guarantees of de-identification and appropriate research uses.

Harmonizing existing ethical review practices is a great idea (Nat. Genet. 43, 817, 2011), but this aim may not be rapidly achievable in practice, given the diversity of institutions and stakeholders. The alternative, piloted by Sage Bionetworks and collaborators, is the idea that research participants can contribute their own data under a portable consent (http://weconsent.us/consentform) to a common consented genomics research environment. From there, the de-identified data can be accessed by any researcher who agrees to protect the research subjects and their data under the terms of the consent and to ensure the accessibility of published derivative results. Because the principle of respect for the autonomy of research subjects underpins all informed consent agreements, it should be possible for research subjects to obtain their own data from any properly consented research project and deposit them in this common consented environment.

Obviously, individuals will vary in their motivation for research participation. As this initiative is designed to be useful for both existing research activities and those not yet imagined, the decision to participate requires some deliberation. Many are likely to want to think over the consequences of the free dissemination of information. Yes, you can withdraw your consent at any time, but publications arising from your data, as well as copies and derivatives of your data, will be freely accessible and may be impossible to recall. However, if you withdraw, your data will no longer be accessible from the common consented environment.

In the spirit of the PLC initiative, parallel efforts to improve human medical research should be inspired by the twin principles of respectful protection for individuals who participate in research and equitable access to data by all competing parties. Legislation does not yet exist to protect research subjects against all foreseeable forms of discrimination, including loss of access to insurance or impersonation and defamation. If these two principles are adhered to, the resulting legislation should be appropriate and reasonable. It must not impose further restrictions on research.

The next step in the evolution of preclinical research, namely ensuring an intellectual property framework that does not lock up medical innovations that might otherwise be implemented, will be a huge challenge, but this is greatly mitigated by the PLC approach that separates the common pool of data from the derivative works. The greater usefulness of accessible and properly consented data from enthusiastic and secure research participants should ensure innumerable real innovations that will more than compensate for the lost illusion of control that data hoarding once seemed to afford. 